\title{
A Note on Distances between Interval-Valued Intuitionistic Fuzzy Sets
}

\author{
Lee-Chae Jang ${ }^{1}$, WonJoo Kim ${ }^{1}$ and T. Kim ${ }^{2}$ \\ 1 Department of Mathematics and Computer Science, Konkuk University, Chungju 380-701, Korea \\ 2 Institute of Natural Science, Konkuk University, Chungju 380-701, Korea \\ 3 Division of General Education, Kwangwoon University, Seoul 139-701, Korea
}

\begin{abstract}
Atanassov [1,2] and Szmidt and Kacprzyk[7,8] studied various methods for measuring distances between intuitionistic fuzzy sets. In this paper, we consider interval-valued intuitionistic fuzzy sets and discuss these methods for measuring distances between interval-valued intuitionistic fuzzy sets.
\end{abstract}

Key Words: intuitionistic fuzzy sets, interval-valued intuitionistic fuzzy set, Hamming distance, Euclidean distance.

\section{Introduction}

The theory of fuzzy sets introduced by Zadeh(1965) has been researched in many new approaches and theories, for examples, intuitionistic fuzzy sets, vague groups, intervalvalued fuzzy sets, Type- 2 fuzzy sets, and interval-valued intuitionistic fuzzy sets(see [1-9]).

Atanassov[1,2] and Szmidt and Kacprzyk[7,8] suggested some methods for measuring distances between intuitionistic fuzzy sets that are generalizations of the well known Hamming distance, the Euclidean distance and their normalized counterparts.

Grzegorzewski[3] investigated new methods for measuring distances between intuitionistic fuzzy sets and the proposed new distances are straight forward generations of the well known Hamming distance, Euclidean distance and their normalized counterparts.

In this paper, we define interval-valued intuitionistic fuzzy sets and discuss these methods(cf. [3,7,8]) for measuring distances between interval-valued intuitionistic fuzzy sets which are different new methods defined by Grzegorzewski[3].

\section{Interval-Valued Intuitionistic Fuzzy Sets}

Let $X$ denote a universe of discourse. Then a fuzzy set $A$ in $X$ is defined as a set of ordered pairs

$$
A=\left\{\left(x, m_{A}(x)\right) \mid x \in X\right\}
$$

Manuscript received Jan. 21, 2011; revised Feb. 23, 2011; Accepted Feb. 24, 2011. where $m_{A}: X \rightarrow[0,1]$ is the membership function of $A$ and $m_{A}(x)$ is the grade of belong of $x$ into $A$.

Let $I=[0,1]$ and $[I]=\left\{\bar{a}=\left[a^{-}, a^{+}\right] \mid a^{-} \leq\right.$ $a^{+}$and $\left.a^{-}, a^{+} \in I\right\}$ be the set of all closed subintervals of $I$. Then according to Zadeh's extension principle [19], we can popularize these operations such as the maximum $\vee$ and the minimum $\wedge . \bar{a} \vee \bar{b}=\left[a^{-} \vee b^{-}, a^{+} \vee b^{+}\right]$ and $\bar{a} \wedge \bar{b}=\left[a^{-} \wedge b^{-}, a^{+} \wedge b^{+}\right]$and also $[I]$ has a minimal element $\overline{0}=[0,0]$ and a maximal element $\overline{1}=[1,1]$. Furthermore, let $\bar{a}=\left[a^{-}, a^{+}\right]$and $\bar{b}=\left[b^{-}, b^{+}\right]$, then we have

$$
\begin{gathered}
\bar{a}=\bar{b} \Leftrightarrow a^{-}=b^{-} \text {and } a^{+}=b^{+}, \\
\bar{a} \leq \bar{b} \Leftrightarrow a^{-} \leq b^{-} \text {and } a^{+} \leq b^{+}, \text {and } \\
\bar{a}<\bar{b} \Leftrightarrow \bar{a} \leq \bar{b} \text { and } \bar{a} \neq \bar{b} .
\end{gathered}
$$

An intuitionistic fuzzy set $A$ in $X$ is given by a set of ordered triples

$$
A=\left\{\left(x, \mu_{A}(x), \nu_{A}(x)\right) \mid x \in X\right\}
$$

where $\mu_{A}, \nu_{A}: X \rightarrow I$ are functions such that

$$
0 \leq \mu_{A}+\nu_{A} \leq 1, \forall x \in X
$$

For each $x \in X$, the numbers $\mu_{A}(x)$ and $\nu_{A}(x)$ represent the degree of membership and degree of nonmembership of the element $x \in X$ to $A \subset X$, respectively. For each element $x \in X$, the intuitionistic fuzzy index of $x$ in $A$ defined as follows

$$
\pi_{A}(x)=1-\mu_{A}(x)-\nu_{A}(x)
$$

Now, we define an interval-valued intuitionistic fuzzy set $\bar{A}=\left[A^{-}, A^{+}\right]$in $X$ is given by a set of ordered triples

$$
\mu_{\bar{A}}=\left\{\left(x, \mu_{\bar{A}}, \nu_{\bar{A}}\right) \mid x \in X\right\}
$$


where $\mu_{\bar{A}}=\left[\mu_{A^{-}}, \mu_{A^{+}}\right], \nu_{\bar{A}}=\left[\nu_{A^{-}}, \nu_{A^{+}}\right]: X \rightarrow[I]$ are interval-valued functions such that

$$
\overline{0} \leq \mu_{\bar{A}}+\nu_{\bar{A}} \leq \overline{1}, \quad \forall x \in X
$$

For each $x$ the interval-valued numbers $\mu_{\bar{A}}(x)$ and $\nu_{\bar{A}}(x)$ represent the interval-valued degree of membership and the interval-valued degree of nonmembership of the element $x \in X$ to $\bar{A} \subset X$, respectively.

We will denote a family of intuitionistic fuzzy sets in $X$ by $\operatorname{IFS}(X)$, while $\operatorname{IIFS}(X)$ stands for the family of all interval-valued intuitionistic fuzzy sets in $X$.

For each element $x \in X$, we can define the intervalvalued intuitionistic fuzzy index of $x$ in $\bar{A}$ defined as follows

$$
\pi_{\bar{A}}(x)=\overline{1}-\mu_{\bar{A}}(x)-\nu_{\bar{A}}(x) .
$$

Then we obtain some basic properties of the interval-valued intuitionistic fuzzy sets and the interval-valued intuitionistic fuzzy index.

Theorem 2.1. (1) If $\bar{A}=\left[A^{-}, A^{+}\right]$is an interval-valued intuitionistic fuzzy set, then $A^{-}$and $A^{+}$are intuitionistic fuzzy sets.

(2) If $\pi_{\bar{A}}=\left[\pi_{1}, \pi_{2}\right]$ is the interval-valued intuitionistic fuzzy index of $x$ in $\bar{A}=\left[A^{-}, A^{+}\right]$, then $\pi_{1}$ and $\pi_{2}$ are intuitionistic fuzzy index of $x$ in $A^{+}$and $A^{-}$, respectively.

Proof. (1) If $\bar{A}=\left[A^{-}, A^{+}\right]$is an interval-valued intuitionistic fuzzy set, $\mu_{A^{-}}, \mu_{A^{+}}: X \rightarrow I$ are the degree of membership of $A^{-}$and $A^{+}$, and $\nu_{A^{-}}, \nu_{A^{+}}: X \rightarrow I$ are the degree of nonmembership of $A^{-}$and $A^{+}$. Since $\overline{0} \leq \mu_{\bar{A}}+\nu_{\bar{A}} \leq \overline{1}$ and

$$
\mu_{\bar{A}}+\nu_{\bar{A}}=\left[\mu_{A^{-}}+\nu_{A^{-}}, \mu_{A^{+}}+\nu_{A^{+}}\right],
$$

we have

$$
0 \leq \mu_{A^{-}}+\nu_{A^{-}} \leq 1
$$

and

$$
0 \leq \mu_{A^{+}}+\nu_{A^{+}} \leq 1
$$

Thus, $A^{-}$and $A^{+}$are intuitionistic fuzzy sets.

(2) Let $\pi=\left[\pi_{1}, \pi_{2}\right]$ be the interval-valued intuitionistic fuzzy index of $x$ in $\bar{A}=\left[A^{-}, A^{+}\right]$. Then for each $x \in X$,

$$
\pi_{1}(x)=1-\mu_{A^{+}}-\nu_{A^{+}}
$$

and

$$
\pi_{2}(x)=1-\mu_{A^{-}}-\nu_{A^{-}} .
$$

Thus, $\pi_{1}$ and $\pi_{2}$ are intuitionistic fuzzy indexes of $x$ in $A^{+}$ and $A^{-}$, respectively.

\section{Measuring Distance between Interval-Valued Intuitionistic Fuzzy Sets}

In this section, we want to express numerically the difference between two objects by means of a distance between corresponding interval-valued intuitionistic fuzzy sets which are generalized intuitionistic fuzzy sets.

At first, we define the interval-valued Hamming distance, the interval-valued normalized Hamming distance, the interval-valued Euclidean distance and the intervalvalued normalized Euclidean distance between intervalvalued intuitionistic fuzzy sets as followings:

Definition 3.1. Let $X=\left\{x_{1}, x_{2}, \ldots, x_{n}\right\}$ be the universe of discourse under study.

(1) An interval-valued mapping $D_{1}: \operatorname{IIFS}(X) \times$ $\operatorname{IIFS}(X) \rightarrow[I]$ is called an interval-valued Hamming distance if for $\bar{A}=\left[A^{-}, A^{+}\right], \bar{B}=\left[B^{-}, B^{+}\right] \in$ $\operatorname{IIFS}(X)$

$$
\begin{aligned}
D_{1}(\bar{A}, \bar{B})=\quad & {\left[d_{1}\left(A^{-}, B^{-}\right) \wedge d_{1}\left(A^{+}, B^{+}\right),\right.} \\
& \left.d_{1}\left(A^{-}, B^{-}\right) \vee d_{1}\left(A^{+}, B^{+}\right)\right]
\end{aligned}
$$

where

$$
\begin{gathered}
d_{1}(C, D)=\frac{1}{2} \sum_{i=1}^{n}\left\{\left|\mu_{C}\left(x_{i}\right)-\mu_{D}\left(x_{i}\right)\right|\right. \\
\left.+\left|\nu_{C}\left(x_{i}\right)-\nu_{D}\left(x_{i}\right)\right|\right\}
\end{gathered}
$$

is the Hamming distance of intuitionistic fuzzy sets $C$ and $D$ defined by Atanassov[2].

(2) An interval-valued mapping $D_{2}: \operatorname{IIFS}(X) \times$ $\operatorname{IIFS}(X) \rightarrow[I]$ is called an the interval-valued normalized Hamming distance if for $\bar{A}=\left[A^{-}, A^{+}\right], \bar{B}=$ $\left[B^{-}, B^{+}\right] \in \operatorname{IIFS}(X)$,

$$
\begin{aligned}
D_{2}(\bar{A}, \bar{B})=\quad[ & d_{2}\left(A^{-}, B^{-}\right) \wedge d_{2}\left(A^{+}, B^{+}\right), \\
& \left.d_{2}\left(A^{-}, B^{-}\right) \vee d_{2}\left(A^{+}, B^{+}\right)\right]
\end{aligned}
$$

where

$$
\begin{gathered}
d_{2}(C, D)=\frac{1}{2 n} \sum_{i=1}^{n}\left\{\left|\mu_{C}\left(x_{i}\right)-\mu_{D}\left(x_{i}\right)\right|\right. \\
\left.+\left|\nu_{C}\left(x_{i}\right)-\nu_{D}\left(x_{i}\right)\right|\right\}
\end{gathered}
$$

is the normalized Hamming distance of intuitionistic fuzzy sets $C$ and $D$ defined by Atanassov[2].

(3) An interval-valued mapping $D_{3}: \operatorname{IIFS}(X) \times$ $\operatorname{IIFS}(X) \rightarrow[I]$ is called an interval-valued Euclidean distance if for $\bar{A}=\left[A^{-}, A^{+}\right], \bar{B}=$ $\left[B^{-}, B^{+}\right] \in \operatorname{IIF} S(X)$,

$$
\begin{aligned}
D_{3}(\bar{A}, \bar{B})=\quad & {\left[d_{3}\left(A^{-}, B^{-}\right) \wedge d_{3}\left(A^{+}, B^{+}\right),\right.} \\
& \left.d_{3}\left(A^{-}, B^{-}\right) \vee d_{3}\left(A^{+}, B^{+}\right)\right]
\end{aligned}
$$


where

$$
\begin{gathered}
d_{3}(C, D)=\left(\frac { 1 } { 2 } \sum _ { i = 1 } ^ { n } \left\{\left(\mu_{C}\left(x_{i}\right)-\mu_{D}\left(x_{i}\right)\right)^{2}\right.\right. \\
\left.+\left(\left|\nu_{C}\left(x_{i}\right)-\nu_{D}\left(x_{i}\right)\right|\right)^{2}\right\}
\end{gathered}
$$

is the Euclidean distance of intuitionistic fuzzy sets $C$ and $D$ defined by Atanassov[2].

(4) An interval-valued mapping $D_{4}: \operatorname{IIFS}(X) \times$ $\operatorname{IIFS}(X) \rightarrow[I]$ is called an interval-valued normalized Euclidean distance if for $\bar{A}=\left[A^{-}, A^{+}\right], \bar{B}=$ $\left[B^{-}, B^{+}\right] \in \operatorname{IIFS}(X)$,

$$
\begin{aligned}
D_{4}(\bar{A}, \bar{B})=[ & {\left[d_{4}\left(A^{-}, B^{-}\right) \wedge d_{4}\left(A^{+}, B^{+}\right),\right.} \\
& \left.d_{4}\left(A^{-}, B^{-}\right) \vee d_{4}\left(A^{+}, B^{+}\right)\right]
\end{aligned}
$$

where

$$
\begin{gathered}
d_{4}(C, D)=\left(\frac { 1 } { 2 n } \sum _ { i = 1 } ^ { n } \left\{\left(\mu_{C}\left(x_{i}\right)-\mu_{D}\left(x_{i}\right)\right)^{2}\right.\right. \\
\left.\left.+\left(\left|\nu_{C}\left(x_{i}\right)-\nu_{D}\left(x_{i}\right)\right|\right)^{2}\right\}\right)
\end{gathered}
$$

is the normalized Euclidean distance of intuitionistic fuzzy sets $C$ and $D$ defined by Atanassov[2].

Now, we will define some other interval-valued distance which proposed to take into account the three parameter characterization of intuitionistic fuzzy sets: the intervalvalued degree of membership, the interval-valued degree of the interval-valued degree of nonmembership and the interval-valued intuitionistic fuzzy index.

Definition 3.2. Let $X=\left\{x_{1}, x_{2}, \cdots, x_{n}\right\}$ be the universe of discourse under study.

(1) An interval-valued mapping $D_{1}^{\prime}: \operatorname{IIFS}(X) \times$ $\operatorname{IIFS}(X) \rightarrow[I]$ is called the Hamming distance if for $\bar{A}=\left[A^{-}, A^{+}\right], \bar{B}=\left[B^{-}, B^{+}\right] \in \operatorname{IIFS}(X)$,

$$
\begin{aligned}
D_{1}^{\prime}(\bar{A}, \bar{B})=[ & {\left[d_{1}^{\prime}\left(A^{-}, B^{-}\right) \wedge d_{1}^{\prime}\left(A^{+}, B^{+}\right),\right.} \\
& \left.d_{1}^{\prime}\left(A^{-}, B^{-}\right) \vee d_{1}^{\prime}\left(A^{+}, B^{+}\right)\right]
\end{aligned}
$$

where

$$
\begin{aligned}
d_{1}^{\prime}(C, D) & =\frac{1}{2} \sum_{i=1}^{n}\left\{\left|\mu_{C}\left(x_{i}\right)-\mu_{D}\left(x_{i}\right)\right|\right. \\
& +\left|\nu_{C}\left(x_{i}\right)-\nu_{D}\left(x_{i}\right)\right| \\
& \left.+\left|\pi_{C}\left(x_{i}\right)-\pi_{D}\left(x_{i}\right)\right|\right\}
\end{aligned}
$$

is the Hamming distance of intuitionistic fuzzy sets $C$ and $D$ defined by Szmidit and Kacprzyk[7,8].

(2) An interval-valued mapping $D_{2}^{\prime}: \operatorname{IIFS}(X) \times$ $\operatorname{IIFS}(X) \rightarrow[I]$ is called the normalized Hamming distance if for $\bar{A}=\left[A^{-}, A^{+}\right], \bar{B}=\left[B^{-}, B^{+}\right] \in$ $\operatorname{IIFS}(X)$,

$$
\begin{aligned}
D_{2}^{\prime}(\bar{A}, \bar{B})=[ & {\left[d_{2}^{\prime}\left(A^{-}, B^{-}\right) \wedge d_{2}^{\prime}\left(A^{+}, B^{+}\right),\right.} \\
& \left.d_{2}^{\prime}\left(A^{-}, B^{-}\right) \vee d_{2}^{\prime}\left(A^{+}, B^{+}\right)\right]
\end{aligned}
$$

where

$$
\begin{array}{r}
d_{2}^{\prime}(C, D)=\frac{1}{2 n} \sum_{i=1}^{n}\left\{\left|\mu_{C}\left(x_{i}\right)-\mu_{D}\left(x_{i}\right)\right|\right. \\
+\left|\nu_{C}\left(x_{i}\right)-\nu_{D}\left(x_{i}\right)\right| \\
\left.+\left|\pi_{C}\left(x_{i}\right)-\pi_{D}\left(x_{i}\right)\right|\right\}
\end{array}
$$

is the normalized Hamming distance of intuitionistic fuzzy sets $C$ and $D$ defined by Szmidit and Kacprzyk[7,8].

(3) An interval-valued mapping $D_{3}^{\prime}: \operatorname{IIFS}(X) \times$ $\operatorname{IIFS}(X) \rightarrow[I]$ is called the Euclidean distance if for $\bar{A}=\left[A^{-}, A^{+}\right], \bar{B}=\left[B^{-}, B^{+}\right] \in \operatorname{IIFS}(X)$,

$$
\begin{aligned}
D_{3}^{\prime}(\bar{A}, \bar{B})=[ & {\left[d_{3}^{\prime}\left(A^{-}, B^{-}\right) \wedge d_{3}^{\prime}\left(A^{+}, B^{+}\right),\right.} \\
& \left.d_{3}^{\prime}\left(A^{-}, B^{-}\right) \vee d_{3}^{\prime}\left(A^{+}, B^{+}\right)\right]
\end{aligned}
$$

where

$$
\begin{aligned}
d_{3}^{\prime}(C, D)=( & \frac{1}{2} \sum_{i=1}^{n}\left\{\left(\mu_{C}\left(x_{i}\right)-\mu_{D}\left(x_{i}\right)\right)^{2}\right. \\
& +\left(\left|\nu_{C}\left(x_{i}\right)-\nu_{D}\left(x_{i}\right)\right|\right)^{2} \\
& \left.\left.+\left(\left|\pi_{C}\left(x_{i}\right)-\pi_{D}\left(x_{i}\right)\right|\right)^{2}\right\}\right)^{\frac{1}{2}}
\end{aligned}
$$

is the Euclidean distance of intuitionistic fuzzy sets $C$ and $D$ defined by Szmidit and Kacprzyk[7,8].

(4) An interval-valued mapping $D_{4}^{\prime}: \operatorname{IIFS}(X) \times$ $\operatorname{IIFS}(X) \rightarrow[I]$ is called the normalized Euclidean distance if for $\bar{A}=\left[A^{-}, A^{+}\right], \bar{B}=\left[B^{-}, B^{+}\right] \in$ $\operatorname{IIFS}(X)$,

$$
\begin{aligned}
D_{4}^{\prime}(\bar{A}, \bar{B})= & {\left[d_{4}^{\prime}\left(A^{-}, B^{-}\right) \wedge d_{4}^{\prime}\left(A^{+}, B^{+}\right),\right.} \\
& \left.d_{4}^{\prime}\left(A^{-}, B^{-}\right) \vee d_{4}^{\prime}\left(A^{+}, B^{+}\right)\right]
\end{aligned}
$$

where

$$
\begin{aligned}
d_{4}^{\prime}(C, D)=\left(\frac { 1 } { 2 n } \sum _ { i = 1 } ^ { n } \left\{\left(\mu_{C}\left(x_{i}\right)-\mu_{D}\left(x_{i}\right)\right)^{2}\right.\right. \\
+\left(\left|\nu_{C}\left(x_{i}\right)-\nu_{D}\left(x_{i}\right)\right|\right)^{2} \\
\left.\left.+\left(\left|\pi_{C}\left(x_{i}\right)-\pi_{D}\left(x_{i}\right)\right|\right)^{2}\right\}\right)^{\frac{1}{2}}
\end{aligned}
$$

is the normalized Euclidean distance of intuitionistic fuzzy sets $C$ and $D$ defined by Szmidit and Kacprzyk[7,8].

Theorem 3.3. Let $X$ be a finite universe of discourse and $\left[R^{+}\right]$the class of all closed intervals of $[0, \infty]$. Then the above interval-valued distances $D_{1}, D_{2}, D_{3}, D_{4}$ : $\operatorname{IIFS}(X) \times \operatorname{IIFS}(X) \rightarrow\left[R^{+}\right]$are all metrics.

Proof. We will give the proof only for $D_{1}$. Let $\bar{A}, \bar{B} \in$ $\operatorname{IIFS}(X)$ and $X=\left\{x_{1}, x_{2}, \ldots, x_{n}\right\}$. Clearly, we have

$$
D_{1}(\bar{A}, \bar{B}) \leq \overline{0} .
$$


If $\bar{A}, \bar{B}$, then $A^{-}=B^{-}$and $A^{+}=B^{+}$. Thus

$$
d_{1}\left(A^{-}, B^{-}\right)=0
$$

and

$$
d_{1}\left(A^{+}, B^{+}\right)=0 .
$$

Then we have $D_{1}(\bar{A}, \bar{B})=0$. Conversely, If $D_{1}(\bar{A}, \bar{B})=$ 0 , then we get

$$
d_{1}\left(A^{-}, B^{-}\right) \wedge d_{1}\left(A^{+}, B^{+}\right)=0
$$

and

$$
d_{1}\left(A^{-}, B^{-}\right) \vee d_{1}\left(A^{+}, B^{+}\right)=0 .
$$

Thus $d_{1}\left(A^{-}, B^{-}\right)=0$ and $d_{1}\left(A^{+}, B^{+}\right)=0$. Then we have $A^{-}=B^{-}$and $A^{+}=B^{+}$, that is, $\bar{A}=\bar{B}$. Since $d_{1}$ has the symmetry properties

$$
d_{1}\left(A^{-}, B^{-}\right)=d_{1}\left(B^{-}, A^{-}\right)
$$

and

$$
d_{1}\left(A^{+}, B^{+}\right)=d_{1}\left(B^{+}, A^{+}\right),
$$

we get

$$
D_{1}(\bar{A}, \bar{B})=D_{1}(\bar{B}, \bar{A}) .
$$

Finally, we have to prove the triangle inequality. Let $\bar{A}, \bar{B}, \bar{C} \in \operatorname{IIF} S(X)$. Since $d_{1}$ satisfies the triangle inequality, we obtain the following inequalities:

$$
d_{1}\left(A^{-}, C^{-}\right) \leq d_{1}\left(A^{-}, B^{-}\right)+d_{1}\left(B^{-}, C^{-}\right)
$$

and

$$
d_{1}\left(A^{+}, C^{+}\right) \leq d_{1}\left(A^{+}, B^{+}\right)+d_{1}\left(B^{+}, C^{+}\right) .
$$

Thus, if $d_{1}\left(A^{-}, C^{-}\right) \leq d_{1}\left(A^{+}, C^{+}\right)$, then,

$$
\begin{gathered}
D_{1}(\bar{A}, \bar{C})=\left[d_{1}\left(A^{-}, C^{-}\right), d_{1}\left(A^{+}, C^{+}\right)\right] \\
\leq\left[d_{1}\left(A^{-}, B^{-}\right)+d_{1}\left(B^{-}, C^{-}\right),\right. \\
\left.d_{1}\left(A^{+}, B^{+}\right)+d_{1}\left(B^{+}, C^{+}\right)\right] \\
=D_{1}(\bar{A}, \bar{B})+D_{1}(\bar{B}, \bar{C}) .
\end{gathered}
$$

and if $d_{1}\left(A^{-}, C^{-}\right)>d_{1}\left(A^{+}, C^{+}\right)$, then,

$$
\begin{gathered}
D_{1}(\bar{A}, \bar{C})=\left[d_{1}\left(A^{+}, C^{+}\right), d_{1}\left(A^{-}, C^{-}\right)\right] \\
\leq\left[d_{1}\left(A^{+}, B^{+}\right)+d_{1}\left(B^{+}, C^{+}\right),\right. \\
\left.d_{1}\left(A^{-}, B^{-}\right)+d_{1}\left(B^{-}, C^{-}\right)\right] \\
=D_{1}(\bar{A}, \bar{B})+D_{1}(\bar{B}, \bar{C}) .
\end{gathered}
$$

Similarly, we have the following theorem without the proof of theorem.

Theorem 3.4. Let $X$ be a finite universe of discourse. Then the above interval-valued distances $D_{1}^{\prime}, D_{2}^{\prime}, D_{3}^{\prime}, D_{4}^{\prime}$ : $\operatorname{IIFS}(X) \times \operatorname{IIFS}(X) \rightarrow\left[R^{+}\right]$are all metrics.

\section{References}

[1] K. T. Atanassov, "Intuitionistic fuzzy sets", Fuzzy Sets and Systems 20 (1986), 87-96.

[2] K. T. Atanassov, Intuitionistic fuzzy sets: Theory and Applications, Physica-Verlag,Wyrzburg, (1999).

[3] P. Grzegorzewski, ”Distances between intuitionistic fuzzy sets and interval-valued fuzzy sets based on the Hausdorff metric", Fuzzy sets and systems 148 (2004), no. 319-328.

[4] L. C. Jang,T. Kim,W. J. Kim, ”A note on some algebraic laws for interval-valued fuzzy quantifies", Proceedings of the Jangjeon Marh. Soc. 13(1) (2010), 39-48.

[5] L. C. Jang,H. M. Kim, T. Kim, "A study on the transitivity of interval-valued inclusion measures", Proceedings of the Jangjeon Math. Soc. 12(3) (2009), 281-287.

[6] L. C. Jang,H. M. Kim, T. Kim, "A note on intervalvalued inclusion measures", Proceedings of the Jangjeon Math. Soc. 12(2) (2009), 157-163.

[7] E. Szmidt and J. Kacprzyk, "On measuring distance between intuitionistic fuzzy sets",Notes IFS 3 (1997), 1-13.

[8] E. Szmidt and J. Kacprzyk, "Distances between intuitionistic fuzzy sets", Fuzzy sets and systems. Proc. Seventh Int. Conf. IMPU 98 (1998), 172-178.

[9] L. A. Zadeh, "Fuzzy sets", Information and Control 8 (1965), 338-353.

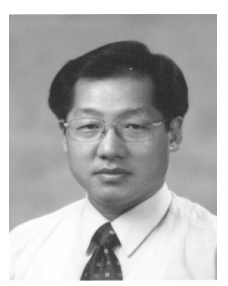

\section{Lee-ChaeJang}

Professor of KonKuk University Research Area: Fuzzy Analysis, Fuzzy Measure, Choquet inetgral, $p$-adic analysis

E-mail: leechae.jang@kku.ac.kr

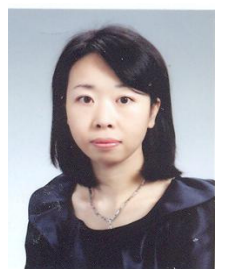

\section{WonJoo Kim}

Institute of Natural Science

Research Area: Fuzzy Analysis, Fuzzy Measure, Choquet inetgral, p-adic analysis

E-mail: piterfan2@naver.com

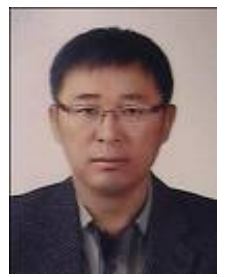

\section{T. Kim}

Professor of Kwangwoon University Research Area: Number Theory, Fuzzy mathematics, $p$-adic Analysis

E-mail: ttkim@kw.ac.kr 